\title{
BMJ Open \\ Values and preferences of women living with HIV who are pregnant, postpartum or considering pregnancy on choice of antiretroviral therapy during pregnancy
}

\author{
Lyubov Lytvyn, ${ }^{1}$ Reed A Siemieniuk, ${ }^{2,3}$ Sophie Dilmitis, ${ }^{4}$ Allyson Ion, ${ }^{5}$ \\ Yaping Chang, ${ }^{2}$ Malgorzata M Bala, ${ }^{6}$ Veena Manja, ${ }^{7}$ Reza Mirza, ${ }^{8}$ \\ Rene Rodriguez-Gutierrez, ${ }^{9,10}$ Hassan Mir, ${ }^{8}$ Laura Banfield, ${ }^{11}$ Per Olav Vandvik, ${ }^{1,12}$ \\ Susan Bewley ${ }^{13}$
}

To cite: Lytvyn L,

Siemieniuk RA, Dilmitis S, et al. Values and preferences of women living with HIV who are pregnant, postpartum or considering pregnancy on choice of antiretroviral therapy during pregnancy. BMJ Open 2017;7:e019023. doi:10.1136/ bmjopen-2017-019023

- Prepublication history and additional material for this paper are available online. To view please visit the journal (http:// dx.doi.org/10.1136/bmjopen2017-019023).

Received 7 August 2017 Accepted 17 August 2017

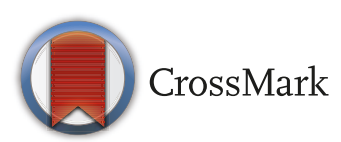

For numbered affiliations see end of article.

Correspondence to

Lyubov Lytvyn;

lytvyn.lyubov@gmail.com

\section{ABSTRACT}

Objective To investigate women's values and preferences regarding antiretroviral therapy (ART) during pregnancy to inform a BMJ Rapid Recommendation.

Setting Primary studies reporting patient-reported outcomes relevant to decision-making regarding ART in any clinical and geographical setting.

Participants Women living with HIV who are pregnant, postpartum or considering pregnancy.

Outcome measures Quantitative measurements and qualitative descriptions of values and preferences in relation to ART during pregnancy. We also included studies on women's reported barriers and facilitators to adherence. We excluded studies correlating objective measures (eg, CD4 count) with adherence, or reporting only outcomes which are not expected to differ between ART alternatives (eg, access to services, knowledge about ART).

Results We included 15 qualitative studies reporting values and preferences about ART in the peripartum period; no study directly studied choice of ART therapy during pregnancy. Six themes emerged: a desire to reduce vertical transmission (nine studies), desire for child to be healthy (five studies), concern about side effects to the child (eight studies), desire for oneself to be healthy (five studies), distress about side effect to oneself (10 studies) and pill burden (two studies). None of the studies weighed the relative importance of these outcomes directly, but pill burden/medication complexity appears to be a lower priority for most women compared with other factors. Overall, the body of evidence was at low risk of bias, with minor limitations.

Conclusions Women who are or may become pregnant and who are considering ART appear to place a high value on both their own and their children's health. Evidence on the relative importance between these values when choosing between ART regimens is uncertain. There is variability in individual values and preferences among women. This highlights the importance of an individualised women-centred approach, such as shared decisionmaking when choosing between ART alternatives. Trial registration number International Prospective Register of Systematic Reviews:CRD42017057157.

\section{Strengths and limitations of this study}

- This is the first systematic synthesis of womenreported factors important for shared decisionmaking regarding antiretroviral therapy (ART) during pregnancy.

- Identified themes were consistent with what women on the linked Rapid Recommendation guideline panel considered most important.

- Design and reporting was variable between studies, and it is challenging to separate factors that may influence the choice of ART regimen-personal, community and health service issues are often intertwined.

- In addition to the relative importance of each factor, values and preferences were quite variable between women, highlighting the importance of shared decision-making.

\section{INTRODUCTION}

Antiretroviral therapy (ART) has transformed the health of persons living with HIV. HIV can now be managed as a chronic and stable condition rather than a progressive disease. ${ }^{1}$ Clinical practice guidelines currently recommend combination ART (cART), including a 'backbone' of two nucleoside or nucleotide reverse transcriptase inhibitors (NRTIs) with a third antiretroviral for everyone living with HIV. ${ }^{2-5}$ Pregnant women living with HIV can experience healthy pregnancies with a low risk of vertical transmission (below 1\%) if they are offered multifaceted strategies, including cART. ${ }^{6}$ Option $\mathrm{B}+$ is a relatively new innovation, where women are advised to take cART as soon as they test positive and to continue for life. ${ }^{7-9}$ Support for Option $\mathrm{B}+$ is not unanimous: some have raised concerns about harms of cART to mother and infant. ${ }^{10}$ In addition, there are a number 
of individual, community and health system barriers that women, caregivers and healthcare providers experience when initiating and adhering to cART during and after pregnancy. ${ }^{11-14}$ Among these, commonly cited barriers include stigma, inadequate time to deal with a new diagnosis, denial of HIV status, intimate partner violence ${ }^{1516}$ and access to care. ${ }^{11-14}$

Shared decision-making is a patient-centred approach and has been tested in some chronic conditions, including HIV. ${ }^{17-19}$ Influential guidelines have been criticised for emphasising child over maternal health and by taking a 'public health perspective', thus de-emphasising each woman's autonomy to choose the ART regimen that best fits in the particular woman's context. ${ }^{20}$ Suboptimal relationships and communication between healthcare providers and women living with HIV have important consequences on initiation, adherence and retention in antenatal care. ${ }^{11-14} 17$ Informed discussion between women living with HIV and their healthcare providers about the benefits and harms of cART is important, because values and preferences may differ among women and thus influence their decisions to initiate and adhere to therapy.

Evidence from a recent randomised controlled trial 'Promoting Maternal and Infant Survival Everywhere' suggests that two common cART regimens confer similar reduction of vertical transmission risk, but also raised the possibility that the most commonly used NRTI backbone, tenofovir plus emtricitabine, increases the risk of serious adverse pregnancy outcomes compared with zidovudine (AZT) plus lamivudine. ${ }^{21}$ This study triggered a $B M J$ Rapid Recommendation guideline-a $B M J$ series of trustworthy recommendations published in response to potentially practice-changing evidence. ${ }^{22}{ }^{23}$ A linked systematic review of the available literature found low-to-moderate quality evidence that tenofovir plus emtricitabine increases early preterm birth as well as stillbirth and early neonatal mortality compared with the AZT plus lamivudine backbone. ${ }^{24}$ To inform the BMJ Rapid Recommendation on cART regimens in pregnancy, we performed this systematic review to explore how women living with HIV value possible benefits and harms of ART.

\section{METHODS}

We followed the Meta-analysis of Observational Studies in Epidemiology (MOOSE) reporting guidelines (see online supplementary appendix 1). The study protocol is registered in International Prospective Register of Systematic Reviews: CRD42017057157 (online supplementary appendix 2).

\section{Eligibility criteria}

We considered eligible both quantitative and qualitative studies from any setting and location, enrolling women living with HIV of childbearing age (15-50 years), who were diagnosed at any time, that evaluated the initiation, adherence or change in ART regimen during pregnancy (for any duration, eg, short-course prophylaxis or cART). Included studies must have reported on the values and preferences of women considering or discussing ART during pregnancy. However, women did not have to be pregnant at the time of the study. Studies reporting the following outcomes were eligible: (1) health state value studies, (2) direct choice studies, (3) studies on non-utility measurement of health states and (4) qualitative studies (eg, focus groups, semistructured interviews). There was no language restriction. We focussed exclusively on the women's perspective. We excluded studies that reported overall health-related quality of life, non-primary studies and case reports, analyses of data that are not women-reported (eg, database studies), studies on use of intrapartum ART only and studies not discussing ART. Implementation and equity considerations from a societal perspective were not included.

\section{Search strategy}

We searched MEDLINE, EMBASE and PsycINFO from 1 January 2000 to 11 February 2017 using a combination of keywords and MeSH/EMTREE terms for 'HIV' AND 'pregnancy' AND 'antiretroviral therapy' (see online supplementary appendix 3). We also used a search filter for patient values and preferences, including terms related to health behaviours, patient values and patient preferences. ${ }^{25}$ We reviewed the references of systematic reviews on similar topics ${ }^{11-14}$ for other potentially eligible studies. We searched for grey literature online with Google, using the terms 'HIV' and 'pregnant OR pregnancy' and 'preference OR experience'.

\section{Study selection}

Title and abstract as well as full-text screening was done independently and in duplicate by reviewer pairs (LL, MMB, YC). Disagreements were resolved by consensus.

\section{Quality assessment}

All eligible studies reported qualitative outcomes. We used the Critical Appraisal Skills Programme checklist to assess methodological quality of individual qualitative studies. $^{26}$

\section{Data collection and presentation}

Two reviewers independently and in duplicate extracted data using a standardised spreadsheet in Microsoft Excel. The following details were extracted: first author, publication year, number of participants, participant demographics (eg, age, length of time living with HIV, whether they have previously or are currently taking ART and what regimen, current and previous pregnancies, payer (eg, government-funded programme, private insurance/ benefits, clinical trial), cost and other demographic data as provided, such as drug use, sexual orientation, sex trade work, disabilities, co-infections, experience of violence, history of mental health issues, among others) and evaluative methods (eg, standard gamble, survey techniques, and semistructured interview). 
Box 1 Women living with HIV contributing to this systematic review

Three women living with HIV, two of whom had children after being diagnosed with HIV and another who is considering having children in the future, participated in the BMJ Rapid Recommendations panel. The community representatives received personalised training and support to optimise contributions throughout the guideline development process. These women provided substantive feedback to this systematic review protocol and helped guide interpretation of the results.

Reporting across studies were variable, with some study authors reporting mostly direct quotations, and others reporting author-generated interpretations or themes and rarely reporting direct quotations. Data were extracted from qualitative studies using thematic synthesis. ${ }^{27}$ Quotations and descriptions of findings in the abstract and results of studies were considered. We extracted all quotations and author-identified themes that were relevant to taking ART, for example, concern over toxicity of medication and concern about transmission of HIV. We excluded factors that would be similar for either medication regimen, for example, access to services, experiences or concerns about intimate partner violence. Relevant quotations were coded and categorised to identify common themes. Themes were summarised narratively in a table. Quotes that were most illustrative of identified themes were reported in the description of the themes. We also extracted the funding source of the study and authors' conflicts of interests, when reported. We did not pre-specify important subgroups.

\section{Incorporation into BMJ Rapid Recommendations}

In the BMJ Rapid Recommendations project, a panel of clinicians, researchers, methodologists and patient representatives create rapid and trustworthy recommendations $^{22}$ (box 1). To provide the panel with best current evidence on cART treatment alternatives for all patient-important outcomes, a linked systematic review on safety and efficacy was performed that summarised outcomes related to maternal and child health in pregnant women living with HIV or HIV and hepatitis B. ${ }^{24}$ The BMJ Rapid Recommendation panel considered the evidence from the systematic review on relative effects as well as evidence from this review on women's values and preferences to make recommendations using the Grading of Recommendations, Assessment, Development and Evaluation (GRADE) framework. ${ }^{22}{ }^{28}$ In addition, results were used to inform the online consultation decision aids generated from the evidence summary supporting this BMJ Rapid Recommendations, available online through MAGICapp (http:// www.magicapp.org) (box 2).
Box 2 Linked articles in this BMJ Rapid

Recommendations cluster

Siemieniuk et al. ${ }^{23}$ Antiretroviral therapy in pregnant women living with HIV: a clinical practice guideline (co-submitted).

- BMJ Rapid Recommendation article

Siemieniuk et al. ${ }^{24}$ Antiretroviral therapy for pregnant women living

with HIV or hepatitis B: a systematic review. (co-submitted).

- A systematic review of antiretroviral therapies in pregnant women MAGICapp

- Expanded version of the evidence with multilayered recommendations, evidence summaries and decision aids for use on all devices.

https://www.magicapp.org/public/guideline/VLpr5E

\section{RESULTS}

\section{Study characteristics}

From 1596 unique citations, 96 proceeded to full-text review and 15 studies were eligible for inclusion (figure 1 ). All the included studies were qualitative and published in English, although 12 studies were conducted in another language. None of the studies evaluated women's values and preferences when considering different ART regimens. The included studies sought to identify barriers and facilitators to initiating, adhering to, or stopping ART (13 studies), uptake of HIV care and services (one study) and factors influencing fertility decisions in women living with HIV (one study). Five studies did not report a specific ART regimen. Geographic location varied, with the majority being low-income or middle-income countries (11 studies), most often in sub-Saharan Africa (six studies) (table 1). Three studies reported on feasibility and acceptability of Option $\mathrm{B}+.^{7-9}$ Authors rarely specified whether the included quotes from women about taking ARTs were referring to during pregnancy, after birth, or both. Twelve studies were funded by government-related grants and three did not report funding source ${ }^{29-31}$ : none were industry sponsored. Most studies did not report conflicts of interest (11 studies) $;^{8}{ }^{29-36}$ the rest stated authors had no competing interests. ${ }^{737-40}$

\section{Study quality}

Studies were generally of moderate methodological quality (see online supplementary appendix 4). Most included fewer than 50 participants (seven studies). ${ }^{30-34} 3637$ The most common sampling strategy was convenience (eight studies). ${ }^{29-32} \quad 35-37 \quad 40$ Four studies reported response rates; $50 \%$ in one study ${ }^{37}$ and $90 \%$ or more in three others. ${ }^{32} 3338$ No study discussed the relationship between the researcher and participants (reflexivity). Two studies described the researcher conducting the interviews. ${ }^{9} 39$ No study discussed data saturation.

\section{Values and preferences related to outcomes of ART}

We identified six common themes in studies of the values and preferences of pregnant women living with HIV regarding ART (table 2). 


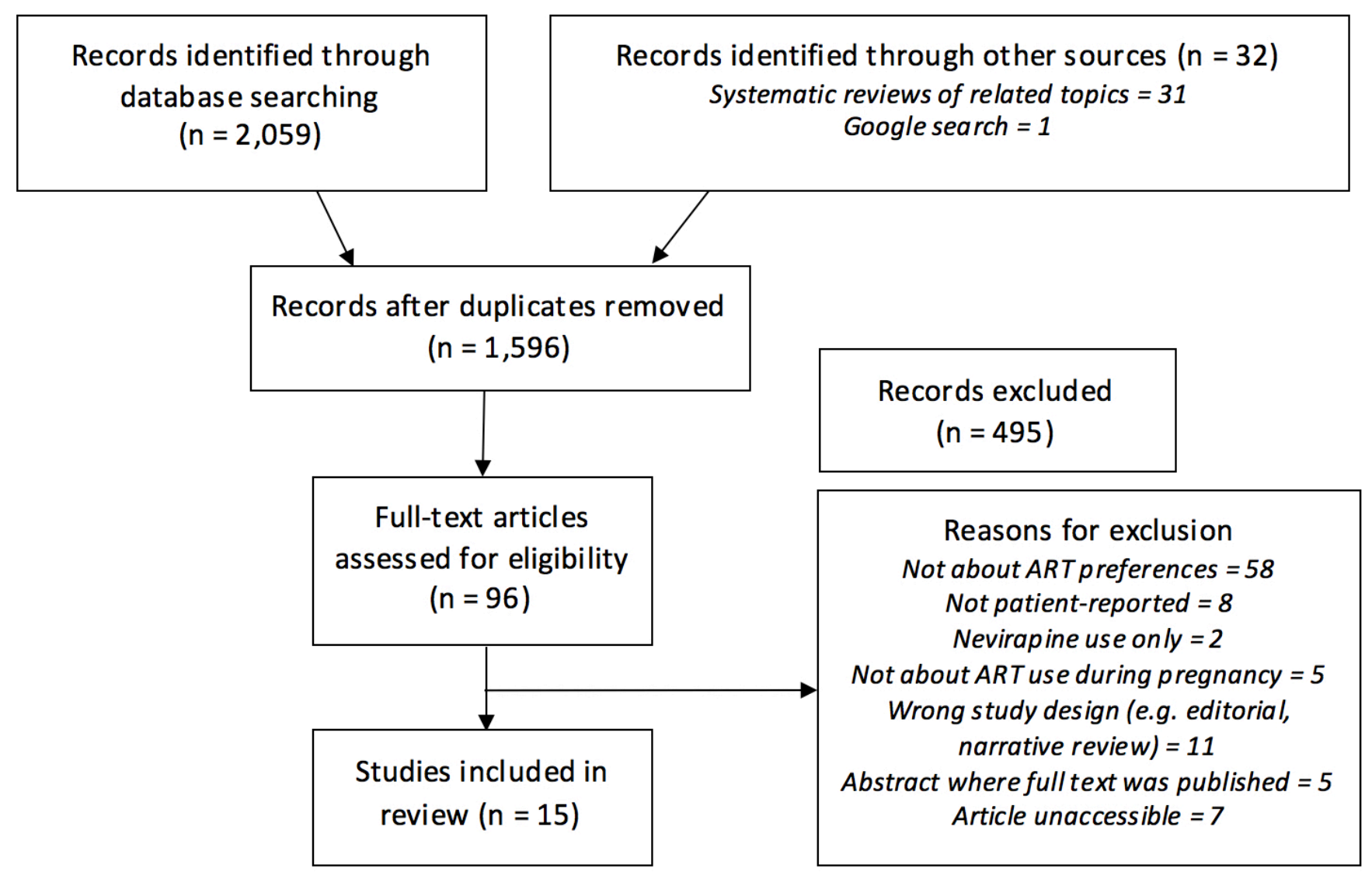

Figure 1 PRISMA study flow diagram. ART, antiretroviral therapy; PRISMA, Preferred Reporting Items for Systematic Reviews and Meta-Analyses.

Desire to reduce vertical transmission risk

Nine studies reported that reducing the risk of vertical transmission is extremely important to most women. Many women expressed a strong desire for having a child without HIV. One woman described "I knew taking them [ARTs] each day was something else, but I really wanted to give birth to a normal child without a disease and that was so precious a thing to me". Women who already had a child without HIV expressed desire to take ARTs again, as one woman said "My last baby is fine, and I would hope that this baby is the same". ${ }^{39}$ Taking ARTs to protect the unborn child appeared universally important, although findings from at least one study suggested that this may not always be the most important factor, as summarised by investigators: "side effects posed a high cost to women, and they had to weigh the future benefit of ART for their child with their immediate health and economic needs". ${ }^{9}$

\section{Desire for child to be healthy}

Several studies reported that a women's concern about their child's health, either before or after birth, was important to them, without specifying exactly what it means to be healthy (five studies). For example, investigators stated "several women expressed that their babies' welfare is what spurred them to take greater responsibility for taking their medications as prescribed [...] their concerns were often focused on health of the unborn child" ${ }^{32}$ Two studies suggested that at least some women had concerns about long-term adverse effects from ART on their child: "concerns for the baby, however, went beyond having a safe and successful delivery and included fears about long-term effects'. ${ }^{36}$ At least one woman expressed her concern that there are "no long-term studies on [the risk of AZT] either, as to what, if anything happens to children from having taken that toxic a drug in their system during their [mothers'] pregnancy". ${ }^{39}$

\section{Concern about ART side effects to the fetus}

Many women are concerned about the possibility of side effects from ART on their fetus (eight studies). This was usually reported by women in general terms, such as "antiretrovirals are quite strong drugs which can ruin the foetus". ${ }^{31}$ One study, however, reported that miscarriage was a specific concern. ${ }^{33}$ Although some women were concerned about the possibility of side effects on their fetus, many still chose to use ART during pregnancy, as summarised by study investigators: "Accepting treatment for the sake of the baby, however, did not free women from worry that it might adversely affect their unborn children". ${ }^{34}$

Desire for oneself to be healthy

In five studies, women revealed a desire to be healthy. For some, this desire was about their general health, as 


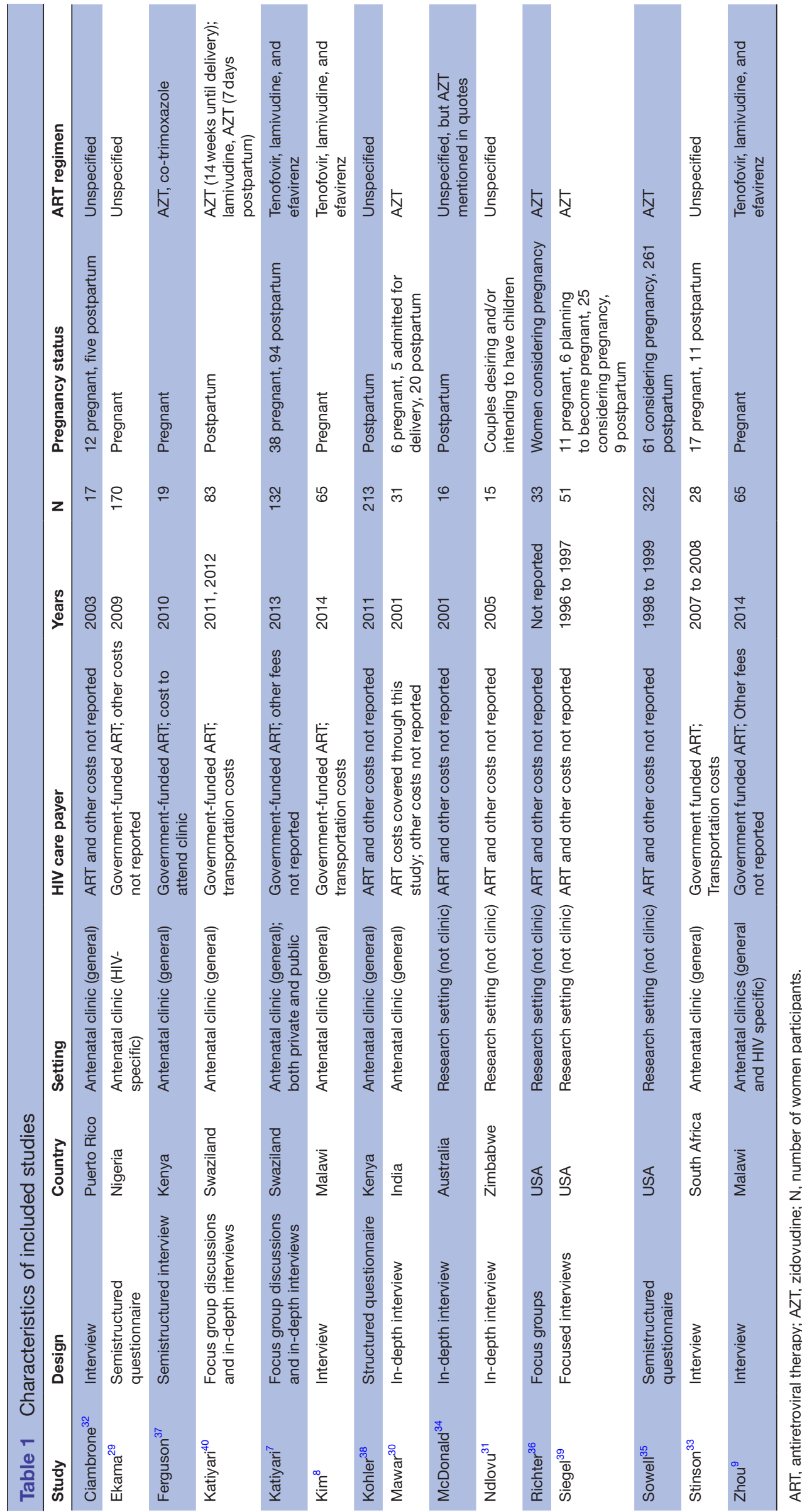


Table 2 Themes addressed in qualitative studies of the values and preferences about antiretroviral therapy in women living with HIV

\begin{tabular}{|c|c|c|c|c|c|c|}
\hline & \multicolumn{3}{|l|}{ Child health } & \multicolumn{2}{|c|}{ Maternal health } & \multirow{2}{*}{$\begin{array}{l}\text { Burden of } \\
\text { treatment } \\
\text { Barrier }\end{array}$} \\
\hline & Facilitator & & Barrier & Facilitator & Barrier & \\
\hline & $\begin{array}{l}\text { Desire to } \\
\text { reduce } \\
\text { transmission }\end{array}$ & $\begin{array}{l}\text { Desire for child } \\
\text { to be healthy }\end{array}$ & $\begin{array}{l}\text { Concern about ART } \\
\text { side effects }\end{array}$ & $\begin{array}{l}\text { Desire to be } \\
\text { healthy }\end{array}$ & $\begin{array}{l}\text { Concern about } \\
\text { ART side effects }\end{array}$ & Pill burden* \\
\hline Ciambrone $2007^{32}$ & & $\checkmark$ & & $\checkmark$ & $\checkmark$ & $\checkmark$ \\
\hline Ekama $2012^{29}$ & $\checkmark$ & $\checkmark$ & & $\checkmark$ & & $\checkmark$ \\
\hline Ferguson $2014^{37}$ & & & $\checkmark$ & & & \\
\hline Katiyari $2016^{40}$ & & & & & $\checkmark$ & \\
\hline Katiyari $2016^{7}$ & & & & $\checkmark$ & & \\
\hline Kim $2016^{8}$ & $\checkmark$ & & & $\checkmark$ & $\checkmark$ & \\
\hline Kohler $2014^{38}$ & & & $\checkmark \dagger$ & & $\checkmark \dagger$ & \\
\hline Mawar $2007^{30}$ & $\checkmark$ & & & & & \\
\hline McDonald $2011^{34}$ & $\checkmark$ & & $\checkmark$ & & $\checkmark$ & \\
\hline Ndlovu $2009^{31}$ & $\checkmark$ & & $\checkmark$ & & & \\
\hline Richter $2002^{36}$ & $\checkmark$ & $\checkmark$ & $\checkmark$ & & $\checkmark$ & \\
\hline Siegel $2001^{39}$ & $\checkmark$ & $\checkmark$ & $\checkmark$ & & $\checkmark$ & \\
\hline Sowell $2001^{35}$ & $\checkmark$ & & $\sqrt{ } \dagger$ & & $\checkmark \dagger$ & \\
\hline Stinson $2012^{33}$ & & $\checkmark$ & $\checkmark$ & & $\checkmark$ & \\
\hline Zhou $2016^{9}$ & $\checkmark$ & & & $\checkmark$ & $\checkmark$ & \\
\hline
\end{tabular}

*Pill burden: reports of forgetting or too busy to take medication, as well as sleeping through medication dosing time, were considered pill burden.

†Authors wrote that women were concerned about harm to mother and/or child but did not provide more detail.

ART, antiretroviral therapy.

one woman stated 'it has given me strength, not getting sick often. Working like other people work and the body isn't weak'. ${ }^{7}$ In another example, health was important to the woman in order to care for the child; "I don't want someone else to have to take care of my child because I can't and that's what worries me the most...it's motivated me to be more responsible". ${ }^{32}$

\section{Concern about ART side effects to oneself}

Side effects were a commonly reported reason for not starting or stopping ARTs (10 studies). For some women, these side effects impacted their ability to provide for their family. One investigator noted: "The effects of ART did not match women's expectation that medicine would treat illnesses and make them feel better. When women compared their health before and after taking ART, they saw a marked decline, often feeling sicker, weaker and unable to carry on with daily tasks". ${ }^{9}$ However, side effects were not always a strong enough deterrent to stop taking ART. Another investigator commented "While most women continued ART despite side effects, some stopped because side effects made them feel worse than before they started treatment". 8
Burden of treatment

None of the studies offered all women multiple ART dosing times per day. Pill burden was indirectly considered from reports of reasons for non-adherence, such as forgetting to take medication, being too busy to take medication due to work or other duties and sleeping through the dose time. ${ }^{29} 32$ Only two studies mentioned these themes, and in neither did pill burden appear to be a major factor when considering ART during pregnancy.

There was no statement from any women about cost being an issue. Cost of drugs was not considered a major factor because ART is supplied free of charge in most programmes from studies in this review (table 1). Some studies report on the cost of using service/transportation instead, which is not expected to differ between ART regimens. ${ }^{83}{ }^{40}$ One study reported that they included women who attended private clinics, but did not report on costs. ${ }^{7}$

\section{DISCUSSION}

Principal findings

This is the first systematic review of the values and preferences of women living with HIV related to the choice of 
ART. Although we did not identify any reports with direct evidence regarding any two ART regimens, there were several studies that were indirectly informative to the study question by reporting key factors for the decision to use or to not use ART around the time of pregnancy. Most studies did not specify the ART regimen considered, and it is unclear whether only one regimen was available for those women, and if they were given any choice.

We identified six factors as being important to pregnant women living with HIV when considering ART: a desire to reduce vertical transmission, a desire for child to be healthy, concern about side effects to the fetus, a desire for oneself to be healthy, concern about side effects and pill burden. There were no formal questions regarding prioritisation, but several quotes suggested that women were balancing the desirable and undesirable consequences of taking ART when making their decisions, for example, reducing vertical transmission and side effects. Many women valued the personal health benefit to taking ARTs during pregnancy, but some found the side effects hard to tolerate and impacted their ability to work and provide for their family. Pill burden was only indirectly identified as a potential concern based on reports of non-adherence relevant to timing of medication regimen in two studies. Because pill burden did not appear to be a major concern in most studies and only indirectly mentioned in two others, it may be less important than the other factors in this population.

\section{Strengths and weaknesses}

Our review has several strengths compared with previously published evidence. First, we followed the MOOSE guidelines, and prospectively registered our protocol. Second, we performed a recent comprehensive search using a validated filter for values and preferences, and did not limit findings to a particular study design nor language of publication. Third, we selected studies, extracted data and conducted quality assessment of primary studies independently and in duplicate. Fourth, the themes we identified in our study were consistent with what the women on the linked Rapid Recommendation guideline panel considered most important for decision-making. ${ }^{23}$

There are some limitations to the current evidence. First, most studies identified additional factors that influenced initiation, adherence and/or stopping ART, which were out of scope for this review. It is challenging to separate factors that may influence the choice of ART regimen, as personal, community and health service issues are often intertwined. ${ }^{811}$ Second, we included three studies that addressed Option $\mathrm{B}+$, where women are advised to start cART as soon as their status is identified during pregnancy, and continue for life. As such, some of the identified factors influencing cART adherence may not have been related to the pregnancy period but rather lifelong therapy. However, identified themes in Option B+ studies appeared to be consistent with studies limited to the pregnancy period. Third, there may be important individual differences, as well as geographic and temporal trends in weighing factors, that were not observed. At least one recent systematic review compared individual, community and healthcare system barriers and facilitators to ART adherence in sub-Saharan Africa before and after 2008, and found that they were consistent. ${ }^{11}$ The identified barriers and facilitators may not be generalisable to all ART regimens. Contextual factors (interpersonal, programme-level) may have a greater influence in some settings compared with others.

\section{Context in relation to the body of literature}

Several related systematic reviews have been published on barriers and facilitators to ART initiation and adherence as well as uptake of perinatal HIV services among women living with HIV who are pregnant or considering pregnancy. ${ }^{11-14}$ Factors that have been associated with better adherence or service uptake include disclosed status, higher education level, knowledge of vertical transmission prevention and ART, care in an urban/district setting rather than a rural one, older age, support in care (by healthcare workers, partners, family and community) and participation in health services. Factors associated with worse adherence or service uptake include denial of HIV, stigma, substance abuse, negative attitudes/ treatment from healthcare workers, scheduling issues (eg, job demands), food and water availability, lost/ stolen medication, under-resourced clinics (long wait times, lack of trained staff, among others), service provider being far from home and cost (transportation, payment for clinic/drugs if not government-provided). Studies provide inconsistent results as to whether religion represents a barrier or a facilitator, which is likely to depend on the individual and the setting. All of these factors were not relevant to a woman's choice of which ART regimen to use after having already decided to initiate ART, but are important to the decision of whether or not to start ART and for long-term medication adherence.

\section{Unanswered questions and future research}

We did not identify any studies about women being given the choice of ART alternatives, or about prioritisation of outcomes related to taking ART. For example, none addressed how important once or twice dosing per day is to women. In most reported settings, the major issue affecting ART initiation was access to any treatment, which inherently makes choice between therapeutic alternatives and dose scheduling a lower priority.

No study commented on participant-researcher reflexivity, despite its importance in qualitative research. ${ }^{41}$ Future research should give more consideration to how the interviewer or research team impacts what women report. In addition, subsequent studies should consider sampling participants until data saturation has been reached, and discussing how this was done. $^{42}$ 
Future research should focus on ways to effectively implement shared decision-making in different settings, including high and low resourced settings. It is important to learn when and how to have these conversations, in what context and with what impact on retention to care, in a way that most effectively empowers women, especially with women who are diagnosed during pregnancy.

\section{CONCLUSIONS}

Many women living with HIV probably consider the health of their child as the most important factor for taking ART during pregnancy, but have to balance this with their own health. Pill burden and medication complexity appears to be a lower priority for most women. There was variability in whether women chose to take ART or not and in the factors that women identified as being important to them. An individualised women-centred approach, such as shared decision-making, should be emphasised for empowering women to make choices that best suit their own values and preferences and accommodate into their context.

\section{Author affiliations}

${ }^{1}$ Faculty of Medicine, Institute of Health and Society, University of Oslo, Oslo, Norway

${ }^{2}$ Department of Health Research Methods, Evidence, and Impact, McMaster University, Hamilton, Canada

${ }^{3}$ Department of Medicine, University of Toronto, Toronto, Canada

${ }^{4}$ Women4GlobalFund, Harare, Zimbabwe

${ }^{5}$ School of Social Work, McMaster University, Hamilton, Canada

${ }^{6}$ Department of Hygiene and Dietetics, Jagiellonian University Medical College,

Krakow, Poland

${ }^{7}$ Department of Medicine, Division of Cardiology, VA Western New York Health Care System, Buffalo, New York, USA

${ }^{8}$ Department of Medicine, McMaster University, Hamilton, Canada

'Internal Medicine, University Hospital ‘Dr. José E. González', Monterrey, Mexico

${ }^{10}$ Department of Medicine, Mayo Clinic, Rochester, Minnesota, USA

${ }^{11}$ Health Sciences Library, McMaster University, Hamilton, Canada

${ }^{12}$ Department of Medicine, Innlandet Hospital Trust-division, Gjøvik, Norway

${ }^{13}$ Women's Health Academic Centre, King's College London, London, UK

Contributors LL and RAS developed the idea for the study and design of the search strategy. LL, RAS, SD, Al, LB and POV were involved in the study conception, study protocol and interpretation of results. LL, YC, MMB, VM, RM, RRG and HM reviewed the study protocol, and were involved in screening and data extraction of papers. All authors reviewed data extraction output. LL, RAS and SB drafted the manuscript, which was critically reviewed and approved by all authors. $L L$ is the guarantor.

\section{Competing interests None declared.}

Provenance and peer review Not commissioned; externally peer reviewed.

Data sharing statement № additional data are available.

Open Access This is an Open Access article distributed in accordance with the Creative Commons Attribution Non Commercial (CC BY-NC 4.0) license, which permits others to distribute, remix, adapt, build upon this work non-commercially, and license their derivative works on different terms, provided the original work is properly cited and the use is non-commercial. See: http://creativecommons.org/ licenses/by-nc/4.0/

(c) Article author(s) (or their employer(s) unless otherwise stated in the text of the article) 2017. All rights reserved. No commercial use is permitted unless otherwise expressly granted.

\section{REFERENCES}

1. Deeks SG, Lewin SR, Havlir DV. The end of AIDS: HIV infection as a chronic disease. Lancet 2013;382:1525-33.

2. Organization WH. Consolidated guideline on sexual and reproductive health and rights of women living with HIV: executive summary. 2017.

3. Phanuphak N, Lolekha R, Chokephaibulkit K, et al. Thai national guidelines for the prevention of mother-to-child transmission of HIV: March 2010. 2010.

4. de Ruiter A, Taylor GP, Clayden P, et al. British HIV Association guidelines for the management of HIV infection in pregnant women 2012 (2014 interim review). HIV Med 2014;15(Suppl 4):1-77.

5. Ryom L, Boesecke C, Gisler V, et al. Essentials from the 2015 European AIDS Clinical Society (EACS) guidelines for the treatment of adult HIV-positive persons. HIV Med 2016;17:83-8.

6. Townsend CL, Byrne L, Cortina-Borja M, et al. Earlier initiation of ART and further decline in mother-to-child HIV transmission rates, 20002011. AIDS 2014;28:1049-57.

7. Katirayi L, Namadingo H, Phiri M, et al. HIV-positive pregnant and postpartum women's perspectives about Option B+ in Malawi: a qualitative study. J Int AIDS Soc 2016;19.

8. Kim MH, Zhou A, Mazenga A, et al. Why did I stop? Barriers and facilitators to uptake and adherence to ART in Option B+ HIV care in Lilongwe, Malawi. PLoS One 2016;11:e0149527.

9. Zhou A. The uncertainty of treatment: Women's use of HIV treatment as prevention in Malawi. Soc Sci Med 2016;158:52-60.

10. Uthman OA, Nachega JB, Anderson J, et al. Timing of initiation of antiretroviral therapy and adverse pregnancy outcomes: a systematic review and meta-analysis. Lancet HIV 2017;4:e21-30.

11. Gourlay A, Birdthistle I, Mburu G, et al. Barriers and facilitating factors to the uptake of antiretroviral drugs for prevention of motherto-child transmission of HIV in sub-Saharan Africa: a systematic review. J Int AIDS Soc 2013;16:18588.

12. Hodgson I, Plummer ML, Konopka SN, et al. A systematic review of individual and contextual factors affecting ART initiation, adherence, and retention for HIV-infected pregnant and postpartum women. PLoS One 2014;9:e111421.

13. Nachega JB, Uthman OA, Anderson J, et al. Adherence to antiretroviral therapy during and after pregnancy in low-income, middle-income, and high-income countries: a systematic review and meta-analysis. AIDS 2012;26:2039-52.

14. Colvin CJ, Konopka S, Chalker JC, et al. A systematic review of health system barriers and enablers for antiretroviral therapy (ART) for HIV-infected pregnant and postpartum women. PLoS One 2014;9:e108150.

15. Siemieniuk RAC, Krentz HB, Miller P, et al. The clinical implications of high rates of intimate partner violence against HIV-positive women. $J$ Acquir Immune Defic Syndr 2013;64:32-8.

16. Siemieniuk RAC, Krentz HB, Gill MJ. Intimate partner violence and HIV: a review. Curr HIVIAIDS Rep 2013;10:380-9.

17. Gourlay A, Wringe A, Birdthistle I, et al. "It is like that, we didn't understand each other": exploring the influence of patient-provider interactions on prevention of mother-to-child transmission of HIV service use in rural Tanzania. PLoS One 2014;9:e106325.

18. Stiggelbout AM, Van der Weijden T, De Wit MP, et al. Shared decision making: really putting patients at the centre of healthcare. BMJ 2012;344:e256.

19. Fuller SM, Koester KA, Guinness RR, et al. Patients' perceptions and experiences of shared decision-making in primary HIV care clinics. $J$ Assoc Nurses AIDS 2017;28:75-84.

20. Welbourn A. WHO's poor consultation with patients on HIV guidance has denied women choice in drug treatment. BMJ 2014;348:f7601.

21. Fowler MG, Qin M, Fiscus SA, et al. Benefits and risks of antiretroviral therapy for perinatal HIV prevention. N Engl J Med Overseas Ed 2016;375:1726-37.

22. Siemieniuk RA, Agoritsas $\mathrm{T}$, Macdonald $\mathrm{H}$, et al. Introduction to $B M J$ Rapid Recommendations. BMJ 2016:i5191.

23. Siemieniuk RA, Mah Ming J, Guyatt G, et al. Antiretroviral therapy for pregnant women living with HIV. BMJ 2017.

24. Siemieniuk RA, Foroutan F, Mirza R, et al. Antiretroviral therapy for pregnant women living with HIV or hepatitis B: a systematic review. BMJ 2017.

25. Wessels M, Hielkema L, van der Weijden T. How to identify existing literature on patients' knowledge, views, and values: the development of a validated search filter. J Med Libr Assoc 2016;104:320-4.

26. . Critical Appraisal Skills Programme (2017)CASP Qualitative Research Checklist. [online]. http:www.casp-uk.net/checklists (accessed Mar 2017).

27. Thomas J, Harden A. Methods for the thematic synthesis of qualitative research in systematic reviews. BMC Med Res Methodol 2008;8:45. 
28 Guyatt GH, Oxman AD, Vist GE, et al. GRADE: an emerging consensus on rating quality of evidence and strength of recommendations. BMJ 2008;336:924-6.

29 Ekama SO, Herbertson EC, Addeh EJ, et al. Pattern and determinants of antiretroviral drug adherence among Nigerian pregnant women. J Pregnancy 2012;2012:1-6.

30 Mawar N, Joshi PL, Sahay S, et al. Concerns and experiences of women participating in a short-term AZT intervention feasibility study for prevention of HIV transmission from mother-to-child. Cult Health Sex 2007;9:199-207.

31 Ndlovu V. Considering childbearing in the age of highly active antiretroviral therapy (HAART): views of HIV-positive couples. SAHARA J-J Soc Asp H 2009;6:58-68.

32 Ciambrone D, Loewenthal HG, Bazerman LB, et al. Adherence among women with HIV infection in Puerto Rico: the potential use of modified directly observed therapy (MDOT) among pregnant and postpartum women. Women Health 2007;44:61-77.

33 Stinson K, Myer L. Barriers to initiating antiretroviral therapy during pregnancy: a qualitative study of women attending services in Cape Town, South Africa. African Journal of AIDS Research 2012;11:65-73.

34 McDonald K, Kirkman M. HIV-positive women in Australia explain their use and non-use of antiretroviral therapy in preventing motherto-child transmission. AIDS Care 2011;23:578-84.
35 Sowell RL, Phillips KD, Seals BF, et al. HIV-infected women's experiences and beliefs related to AZT therapy during pregnancy. AIDS Patient Care STDS 2001;15:201-9.

36 Richter DL, Sowell RL, Pluto DM. Attitudes toward antiretroviral therapy among African American women. Am J Health Behav 2002;26:25-33.

37 Ferguson L, Grant AD, Lewis J, et al. Linking women who test HIVpositive in pregnancy-related services to HIV care and treatment services in Kenya: a mixed methods prospective cohort study. PLoS One 2014;9:e89764.

38 Kohler PK, Ondenge K, Mills LA, et al. Shame, guilt, and stress: community perceptions of barriers to engaging in prevention of mother to child transmission (PMTCT) programs in western Kenya. AIDS Patient Care STDS 2014:28:643-51.

39 Siegel K, Lekas H-M, Schrimshaw EW, et al. Factors associated with HIV-infected women's use or intention to use AZT during pregnancy. AIDS Education and Prevention 2001:13:189-206.

40 Katirayi L, Chouraya C, Kudiabor K, et al. Lessons learned from the PMTCT program in Swaziland: challenges with accepting lifelong ART for pregnant and lactating women - a qualitative study. BMC Public Health 2016;16:1119.

41 Mays N, Pope C. Assessing quality in qualitative research. BMJ 2000;320:50.

42 Guest G, Bunce A, Johnson L. How many interviews are enough? An experiment with data saturation and variability. Field Methods 2006;18:59-82. 
Correction: Values and preferences of women living with HIV who are pregnant, postpartum or considering pregnancy on choice of antiretroviral therapy during pregnancy

Lytvyn L, Siemieniuk RA, Dilmitis S, et al. Values and preferences of women living with HIV who are pregnant, postpartum or considering pregnancy on choice of antiretroviral therapy during pregnancy. BMJ Open 2017;7:e019023. doi:10.1136/ bmjopen-2017-019023

One of the co-authors, Regina El Dib, was inadvertently missed off the author list. The new author list should read:

Lyubov Lytvyn, Reed A Siemieniuk, Sophie Dilmitis, Allyson Ion, Yaping Chang, Malgorzata M Bala, Veena Manja, Reza Mirza, Rene Rodriguez-Gutierrez, Hassan Mir, Regina El Dib, Laura Banfield, Per Olav Vandvik, Susan Bewley

Regina El Dib's affiliation is "Institute of Science and Technology, UNESPUniversidade Estadual Paulista, São José dos Campos, São Paulo, Brazil”.

The new Contributorship statement should read:

LL and RAS developed the idea for the study and design of the search strategy. LL, RAS, SD, AI, LB and POV were involved in the study conception, study protocol and interpretation of results. LL, YC, MMB, VM, RM, RRG, HM, and RED reviewed the study protocol, and were involved in screening and data extraction of papers. All authors reviewed data extraction output. LL, RAS and SB drafted the manuscript, which was critically reviewed and approved by all authors. LL is the guarantor.

Open Access This is an Open Access article distributed in accordance with the Creative Commons Attribution Non Commercial (CC BY-NC 4.0) license, which permits others to distribute, remix, adapt, build upon this work non-commercially, and license their derivative works on different terms, provided the original work is properly cited and the use is non-commercial. See: http://creativecommons.org/licenses/by-nc/4.0/

(c) Article author(s) (or their employer(s) unless otherwise stated in the text of the article) 2018. All rights reserved. No commercial use is permitted unless otherwise expressly granted.

BMJ Open 2018;8:e019023corr1. doi:10.1136/bmjopen-2017-019023corr1

Check for updates 